\title{
Occluded Objects Recognition Using Multiscale Features and Hopfield Neural Networks
}

\author{
Jiann-Shu Lee, ${ }^{*}$ Chin-Hsing Chen, ${ }^{*}$ Yung-Nien Sun, ${ }^{* *}$ \\ and Guan-Shu Tseng* \\ *Department of Electrical Engineering, **Institute of \\ Information Engineering, \\ National Cheng Kung University, Tainan, Taiwan, R.O.C.
}

\begin{abstract}
A new method to recognize partially visible two-dimensional objects by means of multiscale features and Hopfield neural network is proposed. The Hopfield network is employed to perform global feature matching. Since the network only guarantee to converge to a local optimal state, the matching results heavily depend on the initial network state determined by the extracted features. To acquire more satisfactory initial matching results, a new feature vector, consisting of the multiscale evolution of the extremal position and magnitude of the wavelet transformed contour orientation, is developed. These features contain the contour primitives information in a multiscale manner, hence good initial states can be obtained. The good initiation enables the method to recognize objects of even heavily occluded, that can not be achieved by using the Nasrabadi-Li's method. In addition, to make the matching results more insensitive to the threshold value selection of the network, we replace the step-like thresholding function by a ramp-like one. Experimental results have shown that our method is effective even for noisy occluded objects.
\end{abstract}

\section{Introduction}

The problem of recognizing partially visible objects is of considerable interest in the field of industrial automation. This problem has attracted many researchers [1-3] in the field of computer vision. In this paper, we propose a new approach to deal with this problem. To match features in a global manner, we employ the Hopfield neural network. The Hopfield neural network only guarantees to converge to a local optimal state. If the number of false matches is much larger than the number of correct matches in the initial matching, that is the initial state is away from the optimal state, the network possibly converges to an undesirable state. Therefore, an algorithm with bad initial states, like Nasrabadi-Li [4], tends to fail in the case of heavily occluded objects because the visible feature points are few. To cope with this drawback, features of more effectiveness should be employed. In this paper, we develop a new feature set consisting of the multiscale evolution of the wavelet transformed extremal position and magnitude of the contour orientation. In our previous paper [5], we have 
demonstrated that the evolution at larger scales contains the information about the neighboring primitives. In this paper, we will show that the evolution at smaller scales can even be used to recognize the single primitives including corners and arcs. This means that the evolution conveys the information of the contour primitives in a multiscale manner. In addition, to make the matching results by the Hopfield network less sensitive to the threshold value selection, a ramp-like thresholding function was used to replace the conventional thresholding function which is steplike. Experimental results have shown that our method is effective even for heavily occluded noisy objects.

\section{The Adopted Mother Wavelet}

In this paper, we choose the first derivative of the normalized Gaussian function as the mother wavelet $\psi(x)$,

$$
\psi(x)=-x \exp \left(-x^{2} / 2\right)
$$

The orthogonal wavelets are not used because they usually do not possess mathematical close forms such that analytically mathematical derivation is difficult. In addition, the adopted mother wavelet is the first derivative of a smoothing function, hence its local extrema, namely, the curvature extrema, correspond to the sharper changes of a contour, e.g. corners and arcs.

\section{Single Primitives Recognition Using Wavelet Transformation}

\subsection{Smoothed Orientation Function}

Let $C(t)=(X(t), Y(t))$ represents a regular planar curve where $t$ is the arc length. To improve the orientation resolution, the orientation at point $p_{i}$ is defined as

$$
\phi(i)=\tan ^{-1}\left(\left(Y_{i+q}-Y_{i-q}\right) /\left(X_{i+q}-X_{i-q}\right)\right),
$$

for some $q>1$ to obtain a smoothed version of orientation. In this paper, $q$ is equal to three. This choice causes the orientation profile of a corner to be a ramp-like with ramp-width equal to the smoothing length $S L=2 q+1=7$ instead of a step. As to an arc, the smoothed orientation at $p_{i}$ in Eq. (2) is equivalent to calculating the tangential angle at $p_{i}$ that varies linearly along the arc. Therefore, the smoothed arc orientation profile is still a ramp with the ramp-width equal to $l+S L-1$, where $l$ is the length of the arc. Assuming that $w_{c}$ and $w_{a}$ denote the ramp-width of a corner and an arc with $w_{c}=S L=7$ and $w_{a}=l+S L-1$, respectively. From above, we know that the smoothed orientation profiles of corners and arcs are ramps, and the only difference between them is the ramp-width $w$. 


\subsection{Single Primitives Recognition}

In this section, we will show that the wavelet transform ratio can be used to determine the ramp-width and even angle changes. We define a ramp function $R(x, w)$ as

$$
R(x, w)= \begin{cases}c & \text { if } x<-\frac{w}{2} \\ c+m\left(x+\frac{w}{2}\right) & \text { if }-\frac{w}{2} \leq x \leq \frac{w}{2} . \\ c+m w & \text { if } x>\frac{w}{2}\end{cases}
$$

Performing wavelet transform on $R(x, w)$, we obtain

$$
W R(s, x, w)=m \int_{x-w / 2}^{x+w / 2} \exp \left(-\frac{u^{2}}{2 s^{2}}\right) d u
$$

The modulus maximum of $W R(s, x, w)$ is at $x=0$. The extrema ratio of two scales $s_{a}, s_{b}$, namely, $R\left(s_{a}, s_{b}, w\right)$ is given by

$$
R\left(s_{a}, s_{b}, w\right)=W R\left(s_{a}, 0, w\right) / W R\left(s_{b}, 0, w\right) .
$$

Differentiating $R\left(s_{a}, s_{b}, w\right)$ with respective to $w$, we obtain

$$
\begin{gathered}
R^{\prime}\left(s_{a}, s_{b}, w\right)=\left(\exp \left(-\frac{y^{2}}{2}\right) \int_{0}^{h_{y}} \exp \left(-\frac{u^{2}}{2}\right) d u-k \exp \left(-\frac{k^{2} y^{2}}{2}\right) \int_{0}^{y} \exp \left(-\frac{u^{2}}{2}\right) d u\right) / \\
\left(2\left(\int_{0}^{k y} \exp \left(-\frac{u^{2}}{2}\right) d u\right)^{2}\right)
\end{gathered}
$$

where $y=w /\left(2 s_{a}\right)>0$ and $0<k=s_{a} / s_{b}<1$ (i.e. the scale ratio). Let $N(k, y)$ denote the numerator. By simple computation, we know that $N(k, y)$ is negative, that is, $R^{\prime}\left(s_{a}, s_{b}, w\right)$ is negative, if $w \geq 2 s_{a} \sqrt{\left(2 \ln \left(s_{b} / s_{a}\right)\right) /\left(1-s_{a}^{2} / s_{b}^{2}\right)}$. This result implies that $R\left(s_{a}, s_{b}, w\right)$ is strictly monotonic decreasing for $w \geq 2 s_{a} \sqrt{\left(2 \ln \left(s_{b} / s_{a}\right)\right) /\left(1-s_{a}^{2} / s_{b}^{2}\right)}$. In other words, if a $w$ satisfies this condition a certain $R$ corresponding to a certain $w$ can be used to determine the ramp-width. Because the only difference between a corner and an arc is the value of $w$, this means that the extrema ratio $R$ can not only discriminate a corner from an arc but also can determine the arc length. Since the $w$ is acquired, the other parameter--angle change $m w$ also can be determined easily by applying Eq. (5). This shows that a single primitive can be recognized by using two scales wavelet transform extrema. 


\section{Feature Locating and Feature Selection}

Observing the wavelet transform orientation, we find that most of the local extrema corresponding to the significant structures can appear at least three consecutive scales $s_{1}, s_{2}$ and $s_{3}$ in the test images. Thus, an extremum at $s_{1}$ is regarded as a feature point if it can also appear at $s_{2}$ and $s_{3}$. The feature position is decided at the first scale because the locating capability is better at a smaller scale. We select $s_{1}=2$ for the occluded objects recognition. This selection is not sensitive to noise. In addition, this selection makes the wavelet transform extrema at $s_{1}$ and $s_{2}$ can analyze the single primitive with orientation ramp-width $w \geq 6$. In other words, we can analyze single primitives by using the wavelet transform extrema at $s_{1}$ and $s_{2}$ because the orientation ramp-width of every single primitive is longer than six for a smoothed contour. Hence, we employ the evolution, $\left[W \phi\left(s_{1}, p_{1}\right), W \phi\left(s_{2}, p_{2}\right), W \phi\left(s_{3}, p_{3}\right), p_{2}-p_{1}, p_{3}-p_{2}\right]$ as the contour features, where $W \phi$ is the wavelet transform of the smoothed orientation function $\phi(\cdot)$, and $p_{i}$ denotes the local extremum position at the scale $s_{i}=i s_{1}$. We call the evolution the Integrated Multiscale Feature (IMF).

\section{Feature Matching}

The feature matching task is performed by the Hopfield neural network. The location of the object is then estimated by a least squares fit among the matched feature points. We represent each object by a set of features consisting of IMFs. The energy function used in our method is the same as the Nasrabadi-Lis' [4]

$$
E=-\sum_{i=1}^{M} \sum_{k=1}^{N} \sum_{j=1}^{M} \sum_{i=1}^{N} C_{i k l} V_{i k} V_{j l}+\sum_{i=1}^{M}\left(1-\sum_{k=1}^{N} V_{i k}\right)^{2}+\sum_{k=1}^{N}\left(1-\sum_{i=1}^{M} V_{i k}\right)^{2}
$$

The major component of the compatibility measure $C_{i k l}$ (the synaptic strength) between a neuron in row $i$ column $k$ and in row $j$ column $l$ is defined by

$$
C_{i k j i}=W_{1} F\left(\sum_{y=1}^{5}\left|f_{i, y}-f_{k, y}\right|\right)+W_{2} F\left(\sum_{y=1}^{5}\left|f_{j, y}-f_{l, y}\right|\right)+W_{3} F\left(\left|d_{i, j}-d_{k, l}\right|\right)
$$

where $W_{i}$ for $\mathrm{i}=1,2,3$ are the weighting factors, $f_{i, y}$ and $f_{j, y}$ are the $y$ th elements of the IMFs of the $i$ th and the $j$ th feature points of the model object, $f_{k, l}$ and $f_{k, l}$ are the $y$ th elements of the IMFs of the $k$ th and the $l$ th feature points of the scene, $F(x)$ is the thresholding function, and $d_{x y}$ denotes the distance between the $x$ th and the $y$ th features. The network is updated in the same way as the Nasrabadi-Lis' [4]. The adopted thresholding function $F(x, y)$ in Nasrabadi-Li's algorithm is a step function. There exists an abrupt change at the threshold value in the function. Such a 
discontinuity causes the feature matching sensitive to the threshold value. To avoid such a drawback, we select a ramp-like function $F(x)$ as the thresholding function. Since there is no abrupt change in $F(x)$, the feature matching results of our algorithm are more stable.

\section{Experimental Results}

To test the effectiveness of our method, a noisy image shown in Fig. 1(a) is adopted. The superimposed result of all the recognized objects is shown in Fig. 1(b). The result shows that our method is effective even for heavily occluded objects.

\section{Conclusions}

In this paper, we have proposed a new occluded object recognition method using the multiscale feature vector and the Hopfield neural network. The Hopfield network is used to perform global feature matching. The feature vector consists of the multiscale extrema evolution of position and magnitude of the wavelet transformed contour orientation. The vector contains the information of the contour primitives in a multiscale manner, hence a good initial state of the Hopfield network can be obtained. Such good initiation enables the network to converge to a state which is closer to the global optimum. To make the converging results more insensitive to the threshold value selection of the network, the step-like thresholding function is replaced by a ramp-like one. Experimental results have shown that our method is effective even for noisy objects with heavy occlusion.

\section{References}

1. J. L. Turney, T. N. Mudge and R. A. Voltz, "Recognizing partially occluded parts," IEEE Trans. Pattern Analysis Mach. Intell. PAMI-7, pp. 410-421, 1985.

2. H. C. Liu and M. D. Srinath, "Partial shape classification using contour matching in distance transformation," IEEE Trans. Pattern Analysis Mach. Intell. PAMI12, pp. 1072-1079, 1990.

3. N. Ansari and E. J. Delp, "Partial shape recognition: a landmark-based approach," IEEE Trans. Pattern Anal. Mach. Intell. PAMI-12, pp. 470-483, 1990.

4. Nasser M. Nasrabadi, and Wei Li, "Object recognition by a Hopfield neural network," IEEE Trans. on Syst. Man Cybern. SMC-21, pp. 1523-1535, 1991.

5. J. S. Lee, Y. N. Sun, C. H. Chen, and C. T. Tsai, "Wavelet based corner detection," Pattern Recognition, Vol. 26, No. 6, pp. 853-865, 1993. 




(a)

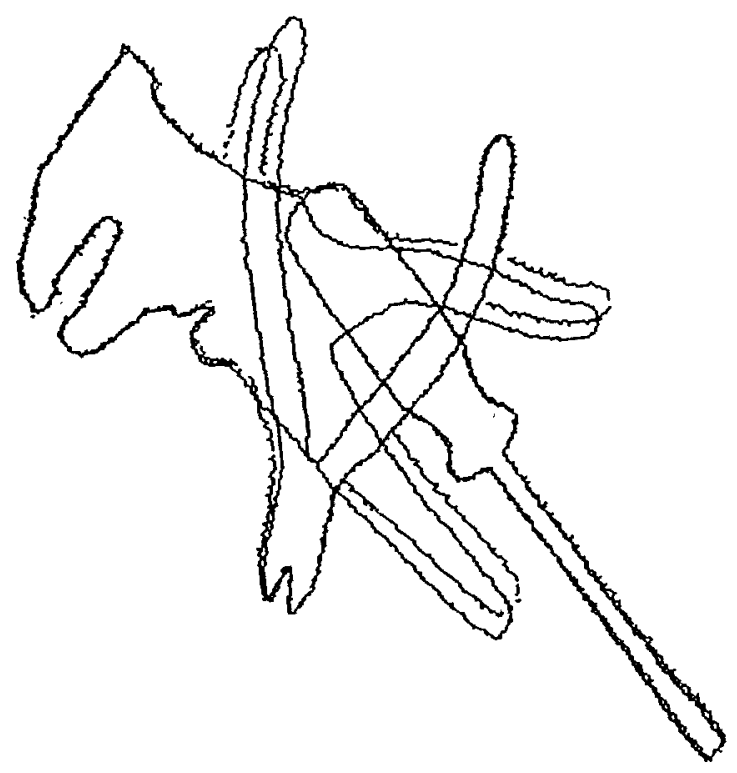

(b)

Fig. 1 (a) The noisy objects and (b) the superimposed result of all the recognized objects. 